- Case Report

\title{
Polycythaemia Vera JAK 2 Mutation in a Patient with Underlying Chronic Obstructive Pulmonary Disease at a Primary Care Setting
}

\author{
Rafidah Mahmud*, Farnaza Ariffin, Punithavathy Shanmuganathan \\ Department of Primary Care Medicine, Faculty of Medicine, University Teknologi MARA (UiTM), Batu Caves, Malaysia
}

The presence of erythrocytosis along with the diagnosis of chronic obstructive pulmonary disease (COPD) may veer a primary care clinician in a busy clinic towards attributing the erythrocytosis to hypoxia secondary to COPD; however, this is not always the case. This case highlights the importance of investigation and the significance not excluding a primary cause in COPD patients with erythrocytosis. A 57-year-old male, presenting with chronic cough, was subsequently diagnosed with COPD clinically and confirmed by spirometry. Erythrocytosis was also incidentally noted. The patient did not have any symptoms of polycythemia or hepatosplenomegaly. Therefore, the erythrocytosis was initially thought to be caused by hypoxia secondary to COPD. However, the JAK2 V617F gene mutation was detected and hence the diagnosis of polycythemia vera was made. Although the erythrocytosis was initially attributed secondary to the underlying pulmonary disease, investigations proved it to be primary in origin. This case report highlights the importance of investigating the underlying cause and to confirm the diagnosis of erythrocytosis as primary and secondary polycythemia differ in their management approach. This will avoid inappropriate diagnosis, treatment, and undesirable outcomes.

Keywords: Polycythemia Vera; Primary Polycythemia; Polycythemia; Janus Kinase 2; Chronic Obstructive Pulmonary Disease

Received: September 13, 2018, Revised: October 16, 2018, Accepted: October 23, 2018

*Corresponding Author: Rafidah Mahmud https://orcid.org/0000-0002-8940-9341

Tel: +60-361264669, Fax: +60-361264888,E-mail: farnaza@uitm.edu.my 


\section{INTRODUCTION}

Polycythemia is defined as an increase in hemoglobin concentration above normal which can be classified into absolute polycythemia or relative polycythemia. Relative polycythemia is the consequences of a reduction in plasma volume which results in hemoconcentration. ${ }^{1)}$ Absolute polycythemia can further be divided into polycythemia vera (PV, primary polycythemia) and secondary polycythemia. ${ }^{1)} \mathrm{PV}$ is caused by an acquired or inherited mutation leading to an abnormality within red blood cell progenitors and the diagnosis is strongly associated with the presence of the JAK2 V617F mutation. ${ }^{2)}$ Secondary polycythemia can be caused by a high secretion of erythropoietin (EPO) in response to chronic hypoxia or as a consequence of an EPOsecreting tumor. ${ }^{3)}$

Theoretically, secondary polycythemia can be attributed to chronic obstructive pulmonary disease (COPD) in response to chronic hypoxia. A study by Ullah et al. ${ }^{4)}$ showed that COPD patients had a significant prevalence of secondary polycythemia. Whilst the prevalence of PV in COPD patients is unknown, the study conducted James et al. ${ }^{5)}$ found that the JAK2 V617F mutation which is strongly associated with PV was not found in secondary polycythemia patients. Patients with polycythemia are at an increased risk of thrombosis, stroke, myocardial infarction, and hemorrhage ${ }^{6)}$ and hence require accurate diagnosis of the underlying causes of polycythemia as primary and secondary polycythemia differ in their management approach.

This case report highlights an occurrence of PV in a chronic smoker patient who was subsequently diagnosed with COPD in a primary care setting.

\section{CASE REPORT}

A 57-year-old male, who is a retired teacher, presented with chronic cough and intermittent shortness of breath which had been present for 1 month. He had productive cough with whitish sputum and reported daily symptoms of early morning sneezing, rhinorrhea, and watery eyes. The patient also complained of dizziness, which occurred almost every day, and was described as generalized light headedness. He denied having fever, hemoptysis, chest pain, abdominal pain, rashes, pruritus, or erythromelalgia. He had no history of childhood asthma or a family history of asthma. The patient had suffered from a myocardial infarction 7 years previously, whereby he had undergone coronary artery bypass surgery and had been stable since. The patient had previously smoked 40 cigarettes a day for a period of 20 years which was now reduced to five cigarettes a day for the last 7 years. The patient reported that he drinks two cans of beer a week. On examination, the patient looked plethoric but was not in respiratory distress. Cardiovascular examination revealed a median sternotomy scar. Respiratory and abdominal examination were unremarkable.

Several investigations were carried out including a chest X-ray which was normal. Spirometry revealed a non-reversible, obstructive picture with a forced expiratory volume in one second (FEV1) of 53\%, a FEV1/forced vital capacity ratio of 68 , and a post bronchodilator responsiveness FEV1 of $9 \%(100 \mathrm{~mL})$. Full blood count (FBC) result showed hemoglobin $21.5 \mathrm{~g} / \mathrm{dL}$, white cell count (WCC) $14 \times 10^{9} / \mathrm{L}$, hematocrit (HCT) $64.5 \%$, and platelets $465 \times 10^{9} / \mathrm{L}$; therefore, he was noted to be polycythemia. Peripheral blood film showed evidence of polycythemia with normochromic, normocytic red blood cells, and leukocytosis (Figure 1). No blast cells were seen. It also revealed presence of thrombocytosis with large and giant platelets.

The patient was subsequently diagnosed with COPD and his symptoms improved after he was started on Ipratropium Bromide/Fenoterol Hydrobromide (metered dose inhaler) and budesonide nasal spray for his allergic rhinitis. He was advised on smoking cessation and was referred to the hematology team for further investigation of the erythrocytosis. The JAK2 V617F gene mutation was subsequently detected, and the diagnosis of PV was made. The patient had an immediate venesection during his first visit to the hematology clinic followed by weekly visits to the day care center for venesection with the aim of lowering his HCT below 45\%. He was also started on Hydroxyurea 500 mg twice a day and continued to take aspirin from his existing medication. After a total of five weekly venesections, the patients FBC parameters showed a HCT of $43.3 \%$, hemoglobin $13.8 \mathrm{~g} / \mathrm{dL}$, WCC $7.94 \times 10^{9} / \mathrm{L}$, and platelets $286 \times 10^{9} / \mathrm{L}$. His dizziness had vastly improved once his FBC parameters had normalized. The patient is currently on monthly follow-ups to monitor his FBC and plan for another venesection if HCT $>45 \%$.

Verbal informed consent for patient information was obtained from the patient himself.

\section{DISCUSSION}

PV is a clonal hematopoietic disorder that belongs to the group of Philadelphia chromosome-negative myeloproliferative neoplasms. ${ }^{7)}$ It is

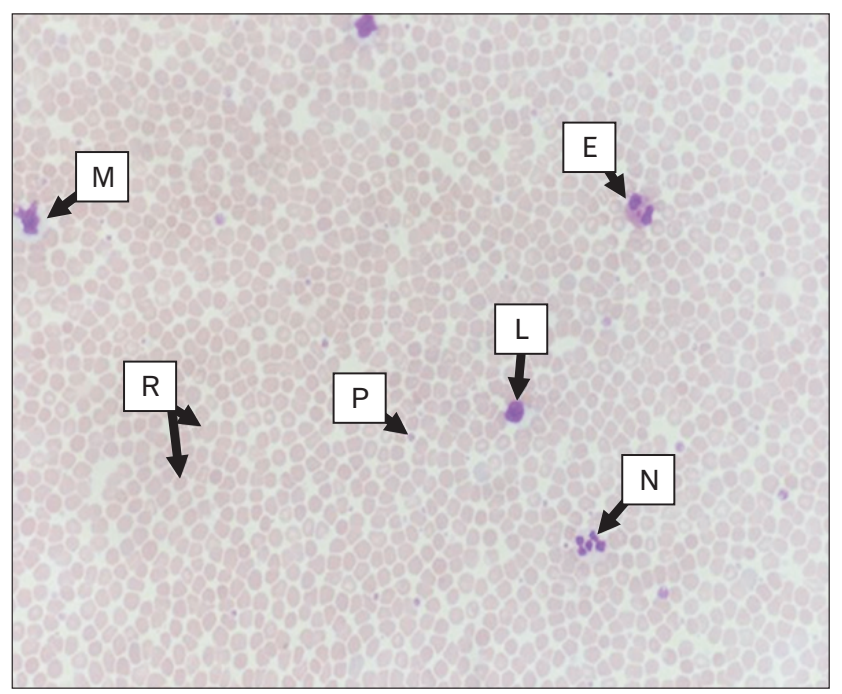

Figure 1. Peripheral blood smear at $40 \times$ magnification demonstrating erythrocytosis. $M$, monocyte; R, red blood cell; P, platelet; L, lymphocyte; N, neutrophil; E, eosinophil. 
often characterized by an increase in trilineage hematopoiesis, erythrocytosis, leukocytosis, thrombocytosis, ${ }^{7}$ and splenomegaly. The diagnosis of PV is strongly associated with the presence of the JAK2 V617F somatic mutation, leading to an abnormality within red blood cell progenitors. ${ }^{2)}$ Studies have found that around $80 \%$ of PV patients have the JAK2 V617F mutation. ${ }^{2,5)}$ Secondary polycythemia is caused by a high secretion of EPO which can be appropriate (in response to chronic hypoxia) or inappropriate (consequence of an EPO-secreting tumor). ${ }^{3)}$ Headaches, dizziness, and blurred vision are common complaints in patients with polycythemia related to an increase in blood viscosity. Pruritus following a warm bath and erythromelalgia are classic signs of PV. A palpable spleen is also found in $40 \%$ of PV cases. Frequently, however, patients are asymptomatic, and elevated hemoglobin is discovered incidentally. ${ }^{1)} \mathrm{A}$ secondary cause of polycythemia is usually suspected if splenomegaly is absent or the high HCT is not accompanied by increases in other cell lines, leukocytosis, or thrombocytosis. ${ }^{7)}$ Serum EPO should be measured if a secondary cause of polycythemia is suspected and levels above normal suggest secondary erythrocytosis. ${ }^{3)}$

In this case, it was initially believed that the erythrocytosis was caused by hypoxia, secondary to COPD. This diagnosis was made as the patient did not portray any symptoms suggestive of PV and his presentation was more typical of a secondary cause. Furthermore, a study by Ullah et al. ${ }^{4)}$ showed that COPD patients have a significant prevalence of secondary polycythemia. This can be explained theoretically as hypoxemia in COPD triggers the production of EPO by the EPO-producing cells of the kidneys, followed by an increased level of red cell mass and HCT. ${ }^{3)}$ However, in this case, the presence of trilineage hematopoiesis from the peripheral blood picture, which showed the presence of erythrocytosis, leukocytosis, and thrombocytosis, indicated that this could be primary in origin. The diagnosis of PV was confirmed by the findings of the JAK2 V617F gene mutation. A study conducted James et al..$^{5}$ found that the JAK2 V617F mutation is strongly associated with PV but is not found in secondary polycythemia patients. They also found that serum EPO levels (either below or at the lower end of normal levels) and presence or absence of the JAK2 V617F mutation had an adequate correlation. Based on the findings of their study, with similar findings by others, ${ }^{2)}$ James et al. ${ }^{5)}$ proposed that the JAK2 V617F should be considered as the first step in the diagnosis of erythrocytosis. In the World Health Organization (WHO) revised criteria ${ }^{8)}$ and the British Committee for Standard in Hematology (BCSH) diagnostic criteria, ${ }^{9}$ a JAK2 mutation is included as one of the major criteria for the diagnosis of $\mathrm{PV}$. According to $\mathrm{BCSH}$, the presence of a JAK2 mutation along with a HCT $>52 \%$ in males and $>48 \%$ in females is sufficient to make the diagnosis of $\mathrm{PV}^{9)}$ The $\mathrm{WHO}$ revised criteria also requires the presence of a JAK2 mutation and raised HCT,

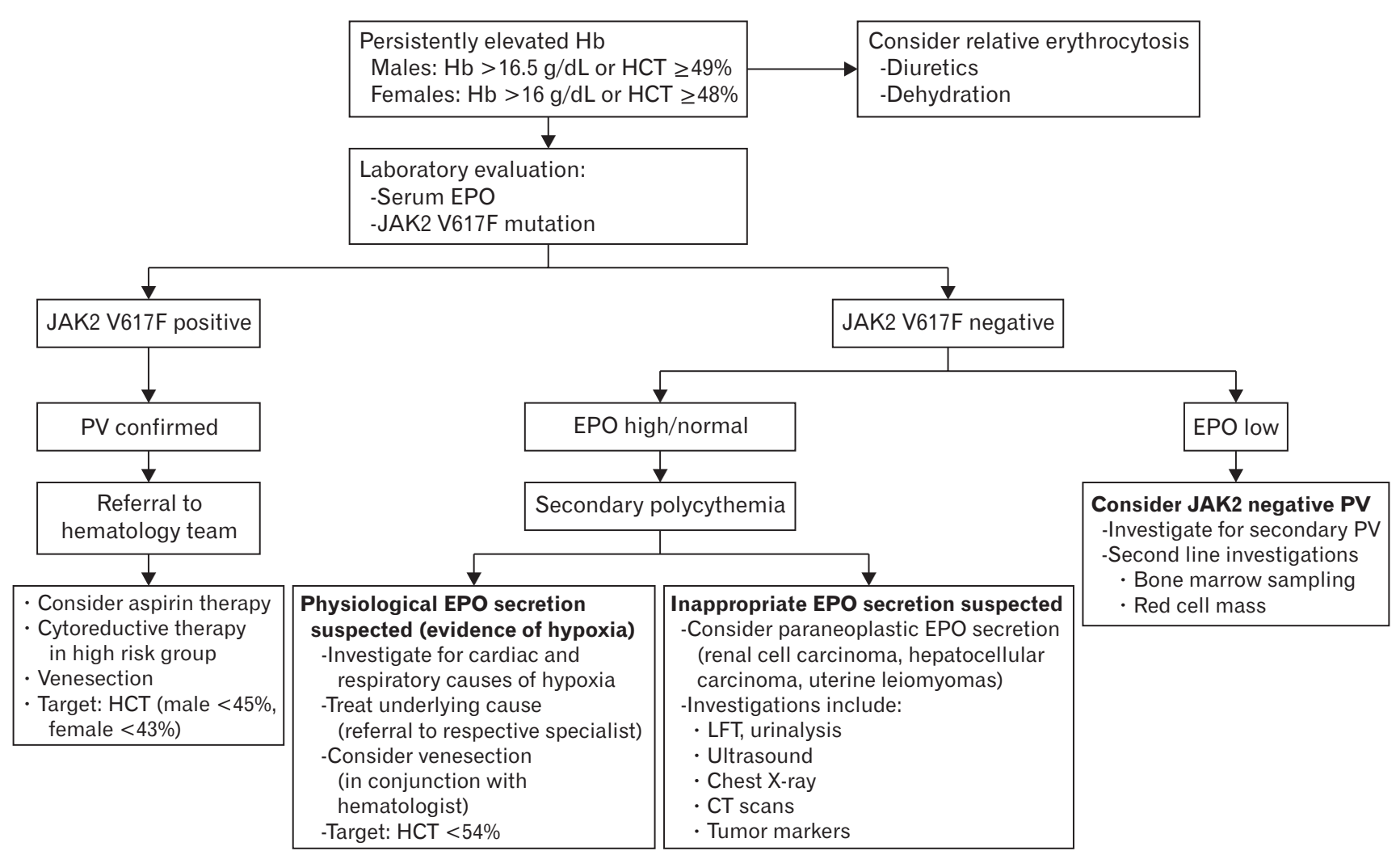

Figure 2. Algorithm for the evaluation of erythrocytosis and management of polycythemia vera. From Tefferi A. Diagnostic approach to the patient with polycythemia [Internet]. Alphen aan den Rijn: UpToDate; 2017 [cited 2018 Jun 28]. Available from: https://www.uptodate.com/contents/diagnostic-approach-to-the-patient-with-polycythemia; ${ }^{11}$ Vyas et al. Oxfordshire Clinical Commissioning Group referral guidelines: polycythaemia. Oxford: Oxfordshire Clinical Commissioning Group; 2012. ${ }^{10)} \mathrm{Hb}$, hemoglobin; HCT, hematocrit; EPO, erythropoietin; PV, polycythemia vera; LFT, liver function test; CT, computed tomography. 
but also requires a minor criteria, for example bone marrow biopsy changes, serum EPO below the normal range, or in vitro endogenous erythroid colony formation. ${ }^{8)}$ It is, however, debatable whether the extra tests are necessary for the diagnosis of PV in the presence of a high HCT and positive JAK2 mutation. ${ }^{3)}$

Polycythemia is associated with an increased risk of thrombosis, stroke, myocardial infarction, and hemorrhage. ${ }^{6)}$ Distinguishing between primary and secondary polycythemia is important in the management of patients with polycythemia as their management approaches are different. Treatment for PV is based on the stratification for risk of thrombosis and includes low-dose aspirin, phlebotomy, and intensive modification of cardiovascular risk factors. High-risk patients also may receive cytoreductive therapy. ${ }^{10)}$ Patients with secondary polycythemia require the removal of underlying causes if possible and limited phlebotomy in the presence of symptoms of an increased blood volume or hyperviscosity. ${ }^{1)}$ In this case, the patient was subsequently followed up by the hematological team for further management of his PV. The patient had several venesections to maintain his HCT below $45 \%$ and prescribed Hydroxyurea $500 \mathrm{mg}$ BD and aspirin. He was also referred to the quit smoking clinic for cessation of smoking and was given medication to control his COPD. Figure 2 below summarizes the evaluation of patients with erythrocytosis and their management approaches. ${ }^{1,10)}$

In conclusion, the management of erythrocytosis is based on the appropriate diagnosis of the underlying pathology and treatment differs between primary and secondary polycythemia. Therefore, we would like to emphasize that the investigation of erythrocytosis, which must entail a careful approach in a primary care setting, should determine the underlying cause to avoid an inappropriate diagnosis, incorrect treatment, and undesirable outcomes.

\section{CONFLICT OF INTEREST}

No potential conflict of interest relevant to this article was reported.

\section{ACKNOWLEDGMENTS}

We would like to thank all clinicians and the patient that provided clinical information

\section{ORCID}

Rafidah Mahmud: https://orcid.org/0000-0002-8940-9341

Farnaza Ariffin: https://orcid.org/0000-0002-7755-9289

Punithavathy Shanmuganathan: https://orcid.org/0000-0002-2147-1245

\section{REFERENCES}

1. Tefferi A. Diagnostic approach to the patient with polycythemia [Internet]. Alphen aan den Rijn: UpToDate; 2017 [cited 2018 Jun 28]. Available from: https://www.uptodate.com/contents/diagnostic-approach-to-the-patient-with-polycythemia.

2. James C, Ugo V, Le Couedic JP, Staerk J, Delhommeau F, Lacout C, et al. A unique clonal JAK2 mutation leading to constitutive signalling causes polycythaemia vera. Nature 2005;434:1144-8.

3. McMullin MF. The classification and diagnosis of erythrocytosis. Int J Lab Hematol 2008;30:447-59.

4. Ullah R, Nasib HA, Iqbal Z, Haq ZU, Ashraf S. Frequency of secondary polycythemia in patients with chronic obstructive pulmonary disease. Pak J Chest Med 2018;24:34-8.

5. James C, Delhommeau F, Marzac C, Teyssandier I, Couedic JP, Giraudier S, et al. Detection of JAK2 V617F as a first intention diagnostic test for erythrocytosis. Leukemia 2006;20:350-3.

6. Elliott MA, Tefferi A. Thrombosis and haemorrhage in polycythaemia vera and essential thrombocythaemia. Br J Haematol 2005;128:275-90.

7. Adamson JW, Fialkow PJ, Murphy S, Prchal JF, Steinmann L. Polycythemia vera: stem-cell and probable clonal origin of the disease. $\mathrm{N}$ Engl J Med 1976;295:913-6.

8. Arber DA, Orazi A, Hasserjian R, Thiele J, Borowitz MJ, Le Beau MM, et al. The 2016 revision to the World Health Organization classification of myeloid neoplasms and acute leukemia. Blood 2016;127:2391-405.

9. McMullin MF, Reilly JT, Campbell P, Bareford D, Green AR, Harrison $\mathrm{CN}$, et al. Amendment to the guideline for diagnosis and investigation of polycythaemia/erythrocytosis. Br J Haematol 2007;138:821-2.

10. Vyas P, Hayes S, Mead AC. Oxfordshire Clinical Commissioning Group referral guidelines: polycythaemia. Oxford: Oxfordshire Clinical Commissioning Group; 2012. 